\title{
O CÂNCER DE MAMA E A SOCIEDADE CIVIL: AS AÇÕES DA FEMAMA NA REGULAMENTAÇÃO TEMPORAL PARA DIAGNÓSTICO E TRATAMENTO DA DOENÇA NO BRASIL ${ }^{1}$
}

\author{
Vanessa Lana ${ }^{2}$ \\ Luiz Antônio Teixeira ${ }^{3}$
}

Recebido em: 24/04/2021

Aprovado em: 26/05/2021

Resumo: A criação das associações de pacientes no Brasil da década de 1990 marcou uma transformação nas formas da sociedade civil demandar ações do poder público em relação à saúde. A criação dessas organizações se relacionou ao novo contexto da saúde no país, surgida com a criação do Sistema Único de Saúde e trouxe para o cenário nacional concepções e práticas de ativismo político e advocacy até então inexistentes nas instituições de pacientes. Este artigo tem como objeto a Federação Brasileira de Instituições Filantrópicas de Apoio à Saúde da Mama, FEMAMA, sua atuação em ações de advocacy, em particular na regulamentação das leis que garantiram marcos temporais para diagnóstico e tratamento do câncer de mama no Brasil na década de 2010. Analisamos a estrutura e organização da instituição e as concepções e atuação na inserção na agenda pública e política de debates da legislação de regulamentação temporal para diagnóstico e tratamento do câncer de mama no setor público de saúde.

Palavras-chave: FEMAMA; Câncer de Mama; Associações de Pacientes; Advocacy; Ativismo político.

\section{CÁNCER DE MAMA Y SOCIEDAD CIVIL: LAS ACCIONES DE FEMAMA EN LA REGULACIÓN TEMPORAL PARA EL DIAGNÓSTICO Y TRATAMIENTO DE LA ENFERMEDAD EN BRASIL}

Resumen: La creación de asociaciones de pacientes en Brasil en la década de 1990 marcó una transformación en las formas en que la sociedad civil exige acciones de las autoridades públicas

\footnotetext{
${ }^{1} \mathrm{O}$ presente trabalho foi realizado com apoio do CNPq, Conselho Nacional de Desenvolvimento Científico e Tecnológico - Brasil (150214/2019-7). Apoio Fundação de Amparo à Pesquisa do Estado do Rio de Janeiro (Faperj) - Programas E-26/202.805/2018 - CNE. E-26/210.115/2016 - Humanidades.

2 Doutora e Pós Doutora em História das Ciências pela Casa de Oswaldo Cruz / FIOCRUZ. Professora do Departamento de História da Universidade Federal de Viçosa e do Mestrado em Patrimônio Cultural, Paisagens e Cidadania. E-mail: vanessalana@ufv.br. ORCID ID: https://orcid.org/0000-0002-1950-1206.

${ }^{3}$ Doutor em História Social. Pesquisador no Departamento de Pesquisa e no Programa de Pós-graduação em História das Ciências e da Saúde, da Casa de Oswaldo Cruz / FIOCRUZ. Professor no Programa de Pós-graduação em Saúde da Criança e da Mulher, do Instituto Fernandes Figueira. Pesquisador de produtividade pelo CNPq. Cientista do Nosso Estado pela Faperj. E-mail: luiztei3@gmail.com. ORCID ID: https://orcid.org/0000-00018871-0928.
} 
en materia de salud. La creación de estas organizaciones estuvo relacionada con el nuevo contexto de salud en el país, que surgió con la creación del Sistema Único de Saúde y trajo al escenario nacional conceptos y prácticas de activismo político y de incidencia que hasta entonces no existían en las instituciones de pacientes. Este artículo tiene como objeto a la Federação Brasileira de Instituições Filantrópicas de Apoio à Saúde da Mama, FEMAMA, su desempeño en acciones de incidencia, en particular en la regulación de leyes que garantizaban plazos para el diagnóstico y tratamiento del cáncer de mama en Brasil en la década de 2010. Analizamos la estructura y organización de la institución y las concepciones y desempeño en la inserción en la agenda pública y política de debates de la legislación de regulación temporal para el diagnóstico y tratamiento del cáncer de mama en el sector público de salud.

Palabras clave: FEMAMA; Cáncer de mama; Asociaciones de pacientes; Abogacía; Activismo político.

\title{
BREAST CANCER AND CIVIL SOCIETY: THE ACTIONS OF FEMAMA IN THE TEMPORAL REGULATION FOR DIAGNOSIS AND TREATMENT OF THE DISEASE IN BRAZIL
}

\begin{abstract}
The creation of patient associations in Brazil in the 1990s marked a transformation in the ways in which civil society demands actions from the public authorities in relation to health. The creation of these organizations was related to the new context of health in the country, which emerged with the creation of the Sistema Único de Saúde and brought to the national scene concepts and practices of political activism and advocacy that did not exist in patient institutions until then. This article has as its object the Federação Brasileira de Instituições Filantrópicas de Apoio à Saúde da Mama, FEMAMA, its performance in advocacy actions, in particular in the regulation of laws that guaranteed time frames for diagnosis and treatment of breast cancer in Brazil in the decade of 2010. We analyzed the structure and organization of the institution and the conceptions and performance in the inclusion in the public agenda and policy of debates of the legislation of temporal regulation for diagnosis and treatment of breast cancer in the public health sector.
\end{abstract}

Keywords: FEMAMA; Breast cancer; Patient Associations; Advocacy; Political activism.

\section{Introdução}

Segundo dados do Instituto Nacional de Câncer José Alencar Gomes da Silva (INCA), o câncer da mama feminina é o tipo mais frequente em todas as regiões do Brasil - não considerando os tumores de pele não melanoma, que apresentam a maior incidência em todo o território. O "Atlas de Mortalidade por Câncer", de 2018, apontou 17.572 óbitos de mulheres por câncer de mama, o que correspondeu a $16,4 \%$ de todas as mortes por neoplasias. Para cada ano do triênio 2020 - 2022 foram estimados 66.280 novos casos, o que indica um risco de cerca de 61,61 novos casos a cada 100 mil mulheres. Dos tipos de câncer mais incidentes no sexo feminino, o câncer de mama representa quase 30\% do total (INCA, 2019). 
A literatura científica aponta que, em países em desenvolvimento, a alta taxa de mortalidade da doença está em muito associada à demora no diagnóstico e tratamento, sendo consenso que quanto mais rápido se institui o tratamento de tumores iniciais, maiores são as chances de cura (BARROS, UEMURA e MACEDO, 2012). A demora no diagnóstico e início do tratamento possibilita o crescimento e evolução do tumor, levando a maiores graus de estadiamento e ampliação dos riscos de metástases (SOUZA, et al, 2015). Dos principais fatores que aumentam a quantidade de indivíduos que atrasam ou não aderem ao tratamento, estão as dificuldades de acesso ao serviço de saúde, o que impacta numa maior mortalidade pela doença (BARROS, UEMURA e MACEDO, 2012).

No âmbito da assistência a pacientes acometidos por câncer de mama são apontados uma série de fatores como possibilidades de reverter os atrasos de diagnóstico e tratamento, tais como: melhor organização dos serviços de saúde, promoção de maior educação da população sobre a doença, implementação de programa de rastreamento, agilidade no encaminhamento dos casos suspeitos para outros níveis de assistência e adequação na realização dos métodos diagnósticos e de tratamento (IDEM). O planejamento de políticas de controle do câncer de mama por meio da detecção precoce é elencado como estratégia fundamental, enquanto assistência. Quanto mais cedo um tumor invasivo é detectado e o tratamento é iniciado, maior a probabilidade de cura (INCA, 2019).

Os estudos que analisaram os intervalos temporais entre o diagnóstico e o início do tratamento do câncer de mama em mulheres no Brasil do início do século XXI apontam que a mortalidade pela doença no pais se apresenta crescente em virtude de retardos no tanto no diagnóstico quanto na implementação da terapêutica adequada. Esse cenário contrasta com o verificado em países desenvolvidos, nos quais é possível observar um aumento da incidência do câncer de mama acompanhado de um declínio nos índices de mortalidade, dado associado à utilização de métodos eficientes de detecção precoce e à oferta de terapêutica adequada (MEDEIROS, et. al, 2015).

O INCA, no documento de consenso de 2004, que orientou diretrizes para a detecção precoce e tratamento do câncer de mama no Brasil, confirmou que o aumento da incidência da doença no país tem sido acompanhado pelo aumento da mortalidade. Tal fato, segundo o documento, pode ser atribuído, principalmente, ao retardamento no diagnóstico e na instituição da terapêutica (BRASIL, 2004). Embora o Brasil tenha instituído uma política nacional visando o diagnóstico do câncer de mama em 1995, com a incorporação da doença ao programa Viva Mulher, as dimensões territoriais, as disparidades sociais e de acesso aos serviços de saúde ainda dificultam a plena concretização de políticas nessa área. 
Nesse estudo buscamos discutir, em perspectiva histórica, a incorporação da demanda pela normatização do tempo de espera pelo diagnóstico e tratamento do câncer de mama e sua transformação em regulamentação legal no Brasil. A análise tem como base a atuação da Federação Brasileira de Instituições Filantrópicas de Apoio à Saúde da Mama, FEMAMA, enquanto associação de pacientes com câncer de mama com foco no ativismo político e advocacy.

A FEMAMA foi criada em 2006. Como primeira proposta de federação de associações de paciente com câncer de mama no país, agregou diferentes instituições de assistência em múltiplas frentes relacionadas à doença. A atuação principal da associação esteve pautada na luta política junto aos poderes públicos e na organização de campanhas de conscientização de direitos e do empoderamento feminino. As ações da FEMAMA caminharam em diversos sentidos. Dentre esses, podemos destacar: estratégias de divulgação para entendimento dos fatores de risco e predisposição à doença; suporte a mulheres em tratamento e iniciativas de conscientização; ampliação da difusão da informação; garantia do acesso e qualidade de mamografias; redução do tempo entre o diagnóstico e o início do tratamento e a união de esforços em prol de uma política nacional contra o câncer de mama - envolvendo governo, classe médica e população em geral (ESTATUTO FEMAMA, 2010).

No primeiro decênio de atuação, a FEMAMA participou, ao lado de suas associadas e junto a outras instituições parceiras, de importantes decisões que mudaram o cenário a favor das pacientes no Brasil. Entre as principais conquistas está a aprovação de um conjunto de leis: Lei 11.664/08, que determinou a realização de mamografias pelo SUS para mulheres a partir dos 40 anos; Lei 12.732/12, que assegurou aos pacientes oncológicos o início do tratamento no SUS em no máximo 60 dias após a inclusão da doença em seu prontuário; Lei 12.880/13, que incluiu tratamentos orais para o câncer na cobertura mínima dos planos de saúde; Lei 12.802/13, que determinou a realização da reconstituição mamária na mesma cirurgia de retirada do tumor, sempre que houver condições médicas; Lei 13.896/2019, que estabelece que os exames relacionados ao diagnóstico de neoplasia maligna sejam realizados no prazo máximo de 30 dias.

Nesse estudo apresentaremos aspectos centrais da trajetória da Federação, tendo como foco as principais campanhas para regulamentação de marcos legais para diagnóstico e início do tratamento para pacientes com câncer de mama no sistema público de saúde. A escolha por trabalhar com essas regulamentações, como elementos para analisar a atuação da FEMAMA, se justifica pelo apontamento na literatura de que o diagnóstico da doença em estágios avançados e a demora no início efetivo do tratamento estão entre os fatores mais relevantes para 
os altos números de mortalidade apresentados no início desse texto, sendo elementos centrais na diminuição das possibilidades de controle e cura.

Para construção desse artigo, dividimos a narrativa em duas grandes partes. Iniciaremos discutindo o conceito de biomedicalização como norte para analisar o surgimento das associações de pacientes e como elemento norteador da análise aqui proposta. Em seguida, discorreremos sobre a formação das associações de pacientes e o contexto de fundação da FEMAMA no Brasil. Na segunda parte, o foco da discussão é a criação e organização da FEMAMA, analisando os preceitos norteadores da Federação e sua atuação em advocacy nos debates e ações para regulamentação temporal do diagnóstico e início do tratamento em câncer de mama no Brasil.

\section{Horizontes conceituais}

Para discutirmos as relações da sociedade com o universo médico e esferas do poder público, em especial a negociação de saberes, tecnologias e práticas médicas, teremos como norte teórico o conceito de biomedicalização. Criado por Adele Clarke e colaboradores (2003), o termo propõe uma atualização do conceito foucaultiano de medicalização, sintetizando o processo de interação entre sociedade e medicina nas últimas décadas. A partir da segunda metade dos anos de 1980, mudanças na organização e nas práticas da biomedicina, empreendidas pela incorporação de novas tecnologias, produziram processos de maior complexidade em nível técnico e social. Desses processos podemos destacar: a privatização de grande parte da pesquisa biomédica, a ampliação de intervenções sociais a partir de tecnologias médicas baseadas na informática, o surgimento de uma nova concepção de vigilância e de saúde relacionada ao risco, o desenvolvimento de novas formas de padronização dos corpos relacionadas ao conhecimento genético, e inovações clínicas na aplicação de medicamentos, testes de diagnóstico e tratamentos (CLARKE, 2003).

No rol de transformações inauguradas com a era da biomedicalização se encontrava a figura dos sujeitos que reivindicavam conhecimentos sobre sua doença e, ao mesmo tempo, buscavam medidas de maximização da saúde através de demandas aos poderes públicos e esferas normativas da sociedade. Os grupos de defesa de pacientes ou movimentos de ativistas em saúde foram centrais a esse processo de busca de conhecimento médico. Segundo os autores que trabalharam com os processos contemporâneos de medicalização e biomedicalização, nas sociedades liberais contemporâneas observou-se uma redução na participação do estado na 
política de regulação da vida, em paralelo a um aumento da participação de entidades regulatórias autônomas e a formação de novas redes de biossociabilidades (CLARKE, 2003; ROSE, 2013). Esse processo, por sua vez, proporcionou um deslocamento dos papeis dos pacientes, que se tornaram cada vez mais consumidores ativos de saúde e sujeitos informados, capazes de agenciar a governabilidade biopolítica (ROSE, 2013). Nesse contexto, floresceram novas formas de ativismo pela saúde relacionadas a padrões culturais e experiências individuais sobre a doença e o adoecimento.

Em relação ao câncer de mama, tendo como base as diferentes formas de compreender e vivenciar o adoecimento e de se organizar socialmente para o seu enfrentamento, estudos recentes discutiram a formação e atuação das associações de pacientes em diferentes contextos e realidades. As análises atentaram tanto para o caráter de associativismo e militância política dessas associações, quanto para as ações com ajuda material e psicológica a mulheres com a doença, incluindo apoio no tratamento e reabilitação (SULIK and ZIERKIEWICZ, 2014).

Maren Klawiter discutiu o desenvolvimento e transformação dos regimes do câncer de mama durante o século XX e seus impactos na emergência de novos sujeitos, solidariedades, grupos e movimentos sociais. Ao analisar os movimentos sociais do câncer de mama nos EUA, a autora apontou para dois regimes de doença: o da medicalização e o da biomedicalização. A ideia de regimes da doença remete à institucionalização de práticas, discursos de autoridade, imagens visuais e publicações sobre como a doença é socialmente construída, gerenciada medicamente, administrada em sua publicização e experenciada no nível da subjetividade. O conceito é tomado como ferramenta de compreensão das relações entre as práticas de biomedicalização em torno do câncer de mama, as formas de subjetividade dos corpos que essas novas práticas produzem e a emergência de novas formas de sociabilidade (KLAWITER, 2008).

No caso do câncer de mama, a transição entre os dois regimes acima apontados se deu por uma série de fatores. Dentre esses fatores estão o desenvolvimento de novas formas de sociabilidade e comunicação entre pacientes, na troca de experiências sobre o adoecimento, relações de suporte, grupos sociais, e a construção de novas identidades e uma maior visibilidade e publicidade da doença. Além desses aspectos, somaram-se questões conjunturais, como a proliferação de procedimentos cirúrgicos, as novas formas de rastreamento, o crescimento de terapias coadjuvantes, a ascensão dos novos discursos de risco, a redefinição dos papeis e responsabilidades de pacientes e médicos e o desenvolvimento de programas de reabilitação que diminuíram o isolamento das pacientes. A emergência de novas formas de medicalização esteve relacionada a novos investimentos e desenvolvimentos em pesquisa 
biomédica e epidemiológica sobre o câncer. A política de rastreamento reinventou o câncer de mama como uma doença de risco invisível e assintomática que requeria contínua vigilância (KLAWITER, 2008).

Os movimentos de pacientes com câncer de mama tiveram influência em mudanças importantes relacionadas à doença, como na defesa de um maior envolvimento da paciente nas decisões sobre as intervenções cirúrgicas, dentro do processo de transformações dos regimes de rastreamento do câncer de mama. Os movimentos ativistas impactaram no monopólio profissional sobre a produção de conhecimento médico, através da defesa da participação ativa das mulheres na aquisição e disseminação da informação científica e no acesso imediato às inovações dos cuidados em saúde (CLARKE, 2003).

O contexto do regime de biomedicalização, que é o contexto da discussão aaqui apresentada, foi marcado, portanto, pela emergência de novos sujeitos e relações sociais da doença, facilitando a formação de novas identidades coletivas, redes de solidariedade e sensibilidades, ou novas formas de "sociabilidade". Essas novas formas estiveram envolvidas com o compartilhamento das experiências de risco, rastreamento, diagnóstico, tratamento e reabilitação, fatores cruciais no desenvolvimento dos movimentos em câncer de mama (KLAWITER, 2008).

O trabalho de Klawiter (2008) mostra-se fundamental para esse artigo ao aproximar as associações de pacientes ao processo mais geral de biomedicalização que implicou em novas relações entre estados, as associações e indústrias biomédicas. Além do preceito de biomedicalização, esse artigo, dentro do rol de atuação das associações de pacientes, destaca os preceitos de ativismo político e advocacy na defesa da regulamentação de marcos temporais de diagnóstico e tratamento da doença, como forma de atuação da FEMAMA no cenário nacional. Para compreender essa atuação, iniciaremos com a contextualização da fundação e organização da Federação.

\section{As associações de pacientes com câncer em perspectiva histórica}

Em estudo sobre ativismo e doenças, Rabeharisoa (2018) apontou a existência de três momentos diferenciados no surgimento de grupos de pacientes, que originaram diferentes modelos de atuação. Nas décadas de 1940 e 1950 surgiram as primeiras associações, como grupos de auto-ajuda, direcionadas às doenças crônicas e visando o compartilhamento de informações e experiências para melhor gestão das enfermidades e seus agravamentos. Nos 
anos 1960 surgiu um segundo modelo formado, sobretudo, por atingidos por doenças causadoras de estigma ou que ocasionavam algum tipo de incapacidade. Esses grupos buscaram, a partir da atuação política, lutar contra o estigma para serem vistos não como doentes mas sim como portadores de uma condição diferenciada de vida. O terceiro modelo de grupos de pacientes, surgido a partir da década de 1980, representou principalmente pacientes com HIV e doenças raras, tendo como principais objetivos a busca por uma melhor compreensão das doenças que os atingiam, a participação nas pesquisas científicas e o de acesso a diferentes tecnologias e terapêuticas (RABEHARISOA, 2018).

No Brasil, podemos observar uma similitude com o terceiro momento acima descrito, na análise sobre a organização da associações de pacientes. No final da década de 1980, o processo de redemocratização do país e a criação de uma nova constituição que transformava a saúde em um direito dos cidadãos e um dever do Estado deu margem ao estabelecimento de novas formas de organização social em torno da saúde. A criação de um sistema mais abrangente, fruto de debates com a sociedade civil, que buscava incorporar as demandas coletivas e a participação social, tornava viável novas formas de demandas por saúde baseadas na ideia de direito. Tais demandas, muitas vezes, se relacionavam às dificuldades do sistema em especial as de financiamentos - no atendimento do direito à saúde, então tornado universal.

Assim, nos anos 1990, no alvorecer do Sistema Único de Saúde, um novo tipo de ativismo relacinado a doenças floresceria. Naquele momento, as mudanças sociais e o exemplo vindo de outras partes do mundo e em especial das associações de pacientes de HIV/Aids, abriram espaço para a criação e atuação de grupos relacionados a diferentes doenças. É importante destacar que o ativismo em relação ao HIV/Aids foi o marco de surgimento de uma nova forma de organização de grupos, que não mais se pautava na busca de criação de instituições filantrópicas ou governamentais ou a auto-ajuda dos membrod. Tratava-se agora de interagir com as instâncias de produção de conhecimentos e demandar políticas e iniciativas estatais que garantissem os direitos dos pacientes (CARNAVALLI, 2021).

Em um contexto, portanto, marcado pela criação de um sistema de saúde de corte universal, com ampliação da cobertura das ações educativas, produção e divulgação de conhecimentos e participação social, foi possível observar uma maior articulação da sociedade civil em torno de questões sobre doenças, como o câncer de mama. Foi a partir desse momento que a organização e atuação das associações de pacientes com câncer de mama se deu de forma efetiva, na atenção integral à mulher e no diálogo com as instâncias públicas na definição de políticas de saúde. Para compreender o contexto do surgimento dessas associações no Brasil, é importante remeter a aspectos históricos das ações e políticas de controle da doença. 
Iniciativas para minorar o problema do câncer de mama no Brasil podem ser observadas desde meados do século XX, quando o conhecimento médico sobre a doença se ampliou e foram criadas instituições para o controle dos cânceres femininos, em especial do colo do útero e da mama. De modo geral, a atenção ao câncer de mama no Brasil até meados do século XX teve como base as instituições públicas ou filantrópicas, em especial os hospitais especializados e os ambulatórios ligados a universidades, com ações baseadas no interesse dos ginecologistas no desenvolvimento de pesquisas em seus campos de atuação (LANA, 2016; 2020). Foi no último quartel do século passado que a visibilidade e iniciativas de controle da doença se ampliassem, impulsionadas pelo desenvolvimento de exames de imagem de mais alta precisão e a constante ampliação da própria incidência do câncer de mama.

De forma semelhante a outras doenças, as mudanças nas abordagens e ações sobre o câncer de mama estiveram condicionadas às negociações de diferentes atores e grupos sociais em torno das tecnologias e práticas para controle e diagnóstico precoce. A partir dos anos 1960, o desenvolvimento de técnicas diagnósticas de visualização do interior das mamas, como o transiluminação e a mastografia, reforçou a possibilidade de observação de tumores bem pequenos. O desenvolvimento do mamógrafo, no início dos anos 1970, tornou mais viável o objetivo de ampliação da detecção precoce em nível populacional, fortalecendo as ações de controle da doença. No Brasil, o primeiro mamógrafo foi importado pelo Instituto Brasileiro de Estudos e Pesquisas em Oncologia e Obstetrícia (Ibepog), ainda na década de 1970. Naquele contexto, instituições públicas, filantrópicas e diversos consultórios privados começaram a adquirir os aparelhos e a treinar médicos para o diagnóstico precoce de tumores mamários, dando início a uma forma de pensar o controle do câncer baseada no conhecimento precoce da doença a partir do exame mamográfico de diagnóstico ou de rastreamento ${ }^{4}$ (PORTO, TEIXEIRA e SILVA, 2013).

Ações mais articuladas de controle do câncer de mama no Brasil foram desenvolvidas a partir dos anos 1980. Em 1983, com o lançamento do Programa de Assistência Integral à Saúde da Mulher (PAISM), o câncer entrava na agenda pública da saúde. O Programa previa uma linha de cuidado que incluía a prevenção e a assistência aos canceres de colo de útero e de mama. Pela primeira vez, o cuidado à mulher ultrapassava a atenção ao ciclo gravídicopuerperal, práticas que historicamente resumiram a ação especifica da saúde pública a esse

\footnotetext{
${ }^{4} \mathrm{O}$ rastreamento mamográfico por exame de imagem é considerado um importante instrumento para a detecção precoce da doença. Consiste em submeter mulheres assintomáticas e sem sinais aparentes do câncer de mama à realização de exame radiológico das mamas, com o objetivo de identificar tumores ocultos, não perceptíveis na inspeção e palpação das glândulas mamárias (SILVA, 2015).
} 
grupo social. As estratégias em relação ao câncer de mama ganharam mais força na década de 1990, quando novas diretrizes do controle da doença, a partir do diagnóstico precoce, foram incorporadas a uma campanha nacional de rastreamento - o "Viva Mulher" (TEIXEIRA, PORTO e NORONHA, 2012).

O referencial normativo para o controle da doença, tendo como base ações de rastreamento, foi definido nacionalmente em 2003, numa reunião de consenso com participação de profissionais do Ministério da Saúde e de Secretarias Estaduais de Saúde, que propiciou o lançamento das "Diretrizes para a Detecção Precoce e Tratamento do Câncer de Mama no Brasil" em 2004 (BRASIL, 2015). Como resultado desse processo, foi criada a "Política Nacional de Atenção Oncológica" e lançado em 2005 o "Plano de Ação para o Controle do Câncer de Mama”, que buscava ampliar a oferta da mamografia de rastreamento e diagnóstica no país (SILVA, 2015). Em 2008, foi criado, o Sistema de Informação do Câncer de Mama (SISMAMA) com o objetivo de gerenciar as ações de investigação precoce e facilitar o acesso aos dados referentes a doença e seu controle, através da otimização da coleta e padronização das informações.

Outra iniciativa governamental relacionada ao câncer de mama foi o lançamento, em 2011, de um plano de ação para fortalecer o Programa Nacional de Cânceres de Colo de Útero e Mama, como parte de um projeto de ampliação da rede de prevenção, diagnóstico e tratamento do câncer. O plano, que se estendeu até 2014, tinha como principal objetivo ampliar o acesso aos exames de rastreamento da doença, em especial a mamografia, e teve como principal objetivo reduzir o tempo entre o diagnóstico e o tratamento, com vistas a diminuir a mortalidade (IDEM).

Em paralelo às ações mais gerais de controle do câncer de mama no Brasil, acima descritas, o início da década de 1990 foi marcado ainda pelo surgimento das associações de pacientes da doença. Até aquele momento, as primeiras associações civis direcionadas ao câncer haviam sido criadas nos anos 1940, sob a denominação de ligas. Tais instituições foram incentivadas pelo Estado Varguista e tinham como principal objetivo arrecadar fundos para a criação e manutenção de hospitais especializados, a realização de campanhas de conscientização sobre a necessidade de diagnóstico precoce e o acolhimento e ajuda aos doentes. Formadas principalmente por médicos e por mulheres da elite das cidades mais ricas, as ligas patrocinaram hospitais filantrópicos de câncer em diversas regiões do pais. A partir do final do governo Vargas, a ação das ligas passou a ser fortemente incentivada pelo Serviço Nacional de Câncer que participava de forma majoritária do financiamento de muitas das instituições por elas mantidas (TEIXEIRA e FONSECA, 2007). 
Nos anos 1990, as mudanças sociais no país abriram espaço para a criação e atuação das associações civis. Uma das primeiras associações foi o Instituto da Mama, IMAMA, fundado em 1993 na cidade de Porto Alegre/RS, com a proposta de promover, manter e reestabelecer a saúde da mama de homens e mulheres em todo o estado do Rio Grande do Sul. A fundação do IMAMA partiu da iniciativa de mulheres então diagnosticadas e em tratamento do câncer de mama, com o propósito de divulgar informação à população gaúcha, enfatizando a importância da detecção precoce da doença para maiores chances de cura, além de qualidade e agilidade em todos os processos da rede de atenção à saúde da mama. $\mathrm{O}$ foco, segundo a fundadora e presidente, a médica Maira Caleffi ${ }^{5}$, era o "bem estar da paciente” (INCA, 2017).

Ao longo de sua trajetória, o Instituto da Mama passou a trabalhar na conscientização das mulheres sobre a importância da detecção precoce do câncer de mama e da necessidade de qualidade e agilidade em todos os processos da rede de atenção à saúde da mama. Num contexto de crescimento, incorporação de outras associações e foco nas pautas políticas relacionadas à doença, com a perspectiva do trabalho em rede, o IMAMA deu origem e foi incorporado à Federação Brasileira de Instituições Filantrópicas de Apoio à Saúde da Mama, FEMAMA, em 2006.

\section{A Federação Brasileira de Instituições Filantrópicas de Apoio à Saúde da Mama - FEMAMA}

A Federação Brasileira de Instituições Filantrópicas de Apoio à Saúde da Mama FEMAMA - foi criada em 22 de julho de 2006, durante o Seminário Visão de Futuro, promovido em São Paulo pelo Instituto da Mama. A FEMAMA surgiu como um desdobramento do "Projeto Te Toca Brasil", que consistia na união de diversas entidades ligadas à causa do câncer de mama para o alinhamento de objetivos que deveriam ser alcançados por meio de planejamento estratégico na busca por melhorias na área da saúde da mama. Essas melhorias estavam relacionadas à diminuição do índice de mortalidade pela doença, à qualidade dos exames e das medicações, a uma maior representatividade no Congresso, entre outros pontos. A criação da Federação tinha como propósito expandir as ações de saúde da mama para outros estados, criando uma grande frente de defesa dos interesses das pacientes (ESTATUTO FEMAMA, 2010).

\footnotetext{
${ }^{5}$ Maira Caleffi nasceu em São Paulo, graduou-se em medicina e farmácia, com doutorado no Guys Hospital Medical School, University of London. Além de presidente do IMAMA e da FEMAMA, ocupa a cadeira de professora titular da Fundação Universidade Federal de Ciências da Saúde de Porto Alegre.
} 
Em seu Estatuto, a FEMAMA se apresentava como uma associação de natureza jurídica de direito privado, sem fins econômicos, beneficente, de caráter educativo, cultural, de saúde e assistência social. A missão da Federação consistia em "articular, propor, conscientizar e defender políticas públicas de atenção à saúde da mama" (IDEM: 2). A associação definiu como metas e estratégias de ação que norteariam os trabalhos: o estímulo ao exercício do direito e deveres de cidadania; a promoção de ações que contribuíssem para o controle do câncer de mama no Brasil em quatro pilares - prevenção, diagnóstico, tratamento e reabilitação; a prestação de serviços de assistência à saúde da mama assim como ações de habilitação e reabilitação de pessoas com deficiências decorrentes de doenças na mama (IDEM).

Uma das especificidades da FEMAMA desde sua organização foi seu caráter federativo, baseado na associação de diferentes instituições que compartilhavam da pauta e nas ações para maior efetividade no diagnóstico e tratamento. Cada instiuição associada mantinha suas particularidades e autonomia de atuação, estando agregadas aos princípios norteadores da Federação. Caberia à Federação o apoio ao desenvolvimento da capacidade de ação política das organizações, associações e indivíduos comprometidos na luta pela saúde mama, preservando a identidade dos movimentos locais regionais, articulando-os em torno de uma agenda nacional compartilhada (ESTATUTO FEMAMA, 2010). Atualmente, a FEMAMA conta com 71 entidades associadas, distribuídas em 17 estados brasileiros mais o Distrito Federal ${ }^{6}$.

Além do princípio de aglutinar instituições que tivessem a saúde da mama como pauta, a própria atuação da FEMAMA foi estabelecida como sendo feita através das entidades filantrópicas e de pessoas físicas envoltas com os propósitos da instituição, além da possibilidade de atuação em conjunto com organizações públicas e privadas nacionais e estrangeiras. A missão proposta pela Associação incluia o objetivo de influenciar a formulação da política pública nacional na saúde para gerar a garantia da universalidade, integralidade e equidade na atenção à saúde, no contexto da saúde integral e dos direitos plenos das mulheres brasileiras (IDEM).

A ideia, portanto, que articulava a proposta de criação da FEMAMA era a possibilidade de atuação na articulação de uma agenda nacional única para influenciar diretamente a organização e implementação de políticas públicas de atenção à saúde da mama no Brasil. Essa proposta tinha por norte o princípio de advocacy. A prática de advocacy traz como pauta uma rediscussão do papel do Estado e da sociedade civil, denotando iniciativas de incidência ou pressão política, de promoção e defesa de uma causa e/ou interesse e de articulações

\footnotetext{
${ }^{6}$ Informações disponíveis em www.femama.org.br. Acesso em: 10 fev. 2021.
} 
mobilizadas por organizações sociais com o objetivo de dar maior visibilidade a determinadas temáticas ou questões no debate público e influenciar a formação de políticas (AVNER, 2002). As ações de advocacy podem se dar em diferentes setores, como no âmbito de atividades de lobby junto aos tomadores de decisão, na participação institucional em conselhos, comitês e fóruns de consulta, fóruns públicos, campanhas cívicas, ou ainda em iniciativas de contestação como manifestações e protestos (ANDREWS, 2004).

A inspiração para fundação da Federação a partir do princípio de advocacy veio da experiência da fundadora e presidente, a mastologista Maira Caleffi, na American Cancer Society, que tinha como base o preceito dos pacientes como protagonistas da busca de soluções para a doença (INCA, 2017). A American Cancer Society (ACS) é uma associação norteamericana, organizada em 1913 por um grupo de cirurgiões com o nome de American Society for the Control of Cancer (ASCC). Em 1944, já firmada no cenário médico estadunidense, a associação foi reorganizada e passou a ser conhecida como ACS, tendo como princípio o fortalecimento da sociedade civil na promoção de ações de advocacy em relação ao controle do câncer (GARDNER, 2006).

As ações em advocacy, no caso do câncer, podem ser pensadas por estratégias de aproximação e relacionamento com os poderes políticos, com gestores, atuação junto a órgãos de regulação como no caso dos medicamentos, envio de informações sobre a rede de atenção oncológica e cobrança de melhorias, participação nos conselhos de saúde e em comitês de discussão de políticas de saúde. Em análise sobre a experiência de advocacy em câncer de mama nos EUA e Canada, Jill Moffett caracterizou o movimento de pacientes como um esforço de base que conferiu voz a mulheres previamente silenciadas, dando maior visibilidade à experiência da doença e seu debate em espaços públicos. O movimento dos grupos de pacientes estaria baseado em três objetivos principais: aumento da consciência sobre o câncer de mama, incluindo suas causas, formas de prevenção, mecanismos de detecção e diagnóstico e tratamentos; suporte emocional, e por vezes financeiro ou legal, para mulheres em vários estágios da doença; e capitação de fundos para pesquisas (MOFFETT, 2015).

Com exceção da captação de fundos para custear pesquisa, as associações de câncer de mama surgidas no Brasil, como a FEMAMA, em grande medida tinham as diretrizes acima como preceitos. Ao se configurar como um perfil de sociedade civil atuante em advocacy com o paciente (INCA, 2017), as práticas e ações da Federação implicaram no desenvolvimento de capacidades de ampliação da base social do movimento e no estabelecimento de alianças com outros espaços, movimentos e agendas de atuação. A promoção e a defesa das ações em advocacy tiveram como objetivos não somente exercer influência sobre uma política pública 
ou sobre o mercado, mas também aumentar a participação cidadã no processo de tomada de decisões, e contribuir para o fortalecimento da sociedade civil, das pacientes com câncer de mama, na busca pela ampliação da cultura democrática.

Na FEMAMA, o foco da prática de advocacy foram as estruturas de poder e os tomadores de decisão que, a partir dos trabalhos técnicos e políticos, interferem diretamente nas condições de saúde da população em geral. A ideia de advocacy englobava a realização de diferentes estratégias para influenciar nas tomadas de decisões sobre as políticas públicas para câncer de mama. As práticas envolveram a busca pelo aumento da conscientização sobre a doença como uma questão social e política, o fortalecimento do perfil de cada associada e da dinâmica do trabalho em rede, principalmente nas iniciativas públicas que remetiam a políticas a nível nacional.

O artigo IX do estatuto da FEMAMA dispõe que a Federação "contribuirá perante as autoridades competentes para a edição, alteração e aperfeiçoamento de textos legais que garantam o direito à prevenção, diagnóstico, tratamento e reabilitação da população no que tange à saúde da mama" (ESTATUTO FEMAMA, 2010). Com base nesses preceitos, os princípios de advocacy propagados pela Federação apontaram, para além dos pontos já destacados, para ações de mobilização social buscando engajamento popular na aprovação de leis, com a convocação da sociedade para participar de consultas públicas sobre diversos temas. Dentre esses, destaca-se a discussão referente ao acesso a diagnóstico e tratamento do câncer de mama, na luta pela conscientização sobre direitos das pacientes, para que essas fossem vigilantes sobre as prerrogativas legais que as beneficiariam.

Sendo o poder legislativo uma das principais frentes do trabalho em advocacy da FEMAMA, como ativismo político, as ações da Federação estiveram pautadas principalmente no envio de informações sobre o câncer de mama, na luta pela construção de uma rede de atenção oncológica, e nos diálogos e pressões para aprovação e regulamentação de projetos de lei relacionados à doença. Desses projetos, destacamos nesse trabalho as leis de regulação temporal do diagnóstico e início do tratamento para pacientes com câncer de mama no sistema público de saúde. Como discutimos no início desse texto, o atraso no diagnóstico e tratamento está relacionado às altas taxas de mortallidade pela doença nos países em desenvolvimento. Assim, atuar nessas questões se mostrava como uma das principais estratégias de garantir os direitos e interesses das pacientes, um dos princípios fundamentais das ações em advocacy.

Posto isso, analisaremos na próxima seção elementos da atuação da FEMAMA na defesa, tramitação e ações para aprovação das leis dos 60 e dos 30 dias: lei 12.732/2012, que assegura a pacientes com diagnóstico de câncer o início do tratamento em até 60 dias, e lei 
13896/2019, que prevê que, mediante suspeita de câncer, a confirmação diagnóstica ocorra na rede pública de saúde em no máximo um mês.

\section{A FEMAMA e a legislação de regulamentação temporal para diagnóstico e tratamento em câncer de mama}

\section{Lei 12.732/2012: "Lei dos 60 dias"}

A lei 12.732/2012 assegurou a pacientes com diagnóstico de câncer o início do tratamento no Sistema Único de Saúde em até 60 dias a contar da detecção da neoplasia maligna, firmada em laudo patológico (BRASIL, 2012). O prazo máximo foi indicado para garantir que o paciente passasse por cirurgia ou iniciasse sessões de tratamento, como quimioterapia ou radioterapia, conforme prescrição médica. Caso este prazo não fosse respeitado, o paciente poderia procurar a Secretaria de Saúde de sua cidade e realizar denúncia junto à ouvidoria do SUS, que seria fiscalizada pelo Ministério da Saúde, ou ainda, recorrer ao judiciário como forma de garantia de seus direitos. A lei buscava diminuir as iniquidades no acesso à rede pública de saúde e otimizar as possibilidades de cura.

A FEMAMA teve ampla participação na articulação política com o poder legislativo para aprovação da referida lei. A ação da Federação se deu através da aliança com a Senadora Ana Amélia Lemos ${ }^{7}$, que favoreceu a participação da FEMAMA nas Audiências Públicas com o Ministério da Saúde. A lei foi sancionada em 2013, sendo que nesse mesmo ano foram publicadas mais duas portarias sobre o tema que geraram uma série de contestações por parte de órgãos da sociedade civil, em particular da FEMAMA. Destas portarias, destaca-se a 876, que alterou a forma de contagem de tempo determinada na lei 12.732,2012, estabelecendo o início do prazo de obrigatoriedade de início do tratamento a partir do registro do laudo do paciente no sistema e não da detecção do câncer.

A Portaria $876 / 13$ contradizia a Lei $12.732 / 12$ ao determinar que prazo de 60 dias se iniciasse a partir do registro do diagnóstico no prontuário do paciente, diferente da postulação da lei que que o início fosse a partir da assinatura do laudo do paciente. A alteração no marco do início da contagem do prazo para tratamento poderia implicar numa menor efetividade do propósito da Lei 12.732, por dificuldades na implementação dos sistemas de registro em diferentes regiões brasileiras. Diante da situação, a FEMAMA articulou um projeto de decreto legislativo, no qual buscava garantir o estabelecido na lei, e fez ainda articulação com a

\footnotetext{
${ }^{7}$ Ana Amélia Lemos foi Senadora pelo Rio Grande do Sul entre 2011 e 2019, pelo Partido Progressistas (PP).
} 
Secretaria da Mulher na Câmara e a Procuradoria da Mulher no Senado para sustar a portaria 876 (FEMAMA, 2020a).

Além da atuação junto ao poder legislativo, em 2013 a FEMAMA lançou duas importantes ações sobre a lei dos 60 dias: a Campanha "O Tempo Corre Contra”, que objetivava pressionar o Congresso para aprovação do projeto da forma que ele havia sido concebido; e uma pesquisa sobre a implementação da Lei 12.732/12 acompanhada de uma campanha de comunicação com denúncias das pacientes sobre o não cumprimento da lei, para cobrar efetividade e transparência por parte do Governo.

A campanha "O Tempo Corre Contra" tinha como principal suporte de divulgação um vídeo com um cronômetro no qual o tempo corria ao contrário, com a informação de que enquanto o tratamento não se iniciava, o "tempo corria contra", tornando-se um fator negativo à paciente. A mensagem principal era de que quanto antes a paciente inicasse seu tratamento, maiores as chances de cura, e, por isso essa deveria ser uma pauta de todos. Desse modo, a campanha colocava a paciente como a grande protagonista da luta contra o câncer de mama, reforçando seus direitos ao pleno atendimento no Sistema Único de Saúde (FEMAMA, 2013).

Para divulgação da campanha, a FEMAMA criou um site com os pontos principais da lei e um espaço para que mulheres relatassem sus histórias de não cumprimento da legislação. O objetivo era que, ao serem coletados 100 relatos, esses seriam reunidos e enviados ao Ministério Público Federal, com o intuito de gerar uma ação civil pública para garantir a total implementação e eftivação da legislação. Além do espaço para a inteiração da paciente, a FEMAMA disponibilizou ainda informações sobre órgãos públicos a serem buscados para reivindicar o pronto atendimento no $\mathrm{SUS}^{8}$.

Interessante observar que além da busca pela garantia dos direitos das pacientes com câncer de mama, através da observação das diretrizes legais, a campanha também visava o fortalecimento do papel do sistema de registros em câncer do Ministério da Saúde. Nesse sentido, o SISCAN ${ }^{9}$ (Sistema de Informação do Câncer) era elencado como um dos pilares para atendimento da Lei. Isso porque, o sistema reunia o histórico do diagnóstico e tratamento dos pacientes oncológicos, e era um instrumento importante para acompanhar o tempo de espera do paciente pelo início do tratamento. O comprometimento do poder publico para agilizar a implantação do SISCAN era um dos pontos defendidos pela Federação.

\footnotetext{
${ }^{8}$ Informações disponíveis em http://www.otempocorrecontra.com.br. Acesso em: 10 jan. 2021.

${ }^{9}$ Instituído pela Portaria MS/GM No 3.394, 30 de Dezembro de 2013, o SISCAN é um software disponibilizado para diferentes níveis de hierarquia: Federal, Estadual e Municipal, que reune o histórico dos pacientes e do tratamento, possibilitando acompanhar o panorama da doença.
} 
No âmbito da campanha "o Tempo Corre Contra", a FEMAMA custeou uma pesquisa sobre a implementação da lei 12.732/2012. O estudo encomendado à Medinsight, foi realizada em 2014, em âmbito nacional, com gestores estaduais de saúde, hospitais e centros de tratamento do Sistema Único de Saúde. O objetivo era traçar o cenário da implementação da lei no país, identificando avanços e dificuldades ${ }^{10}$. O Relatório produzido a partir do estudo elencou dados de estimativas de incidência e mortalidade do câncer de mama no Brasil e apresentou informações sobre a atuação da FEMAMA nos processo de aprovação, regulamentação e fiscalização para ampla implementação da lei. O estudo apontava quatro principais dificuldades enfrentadas pelos serviços e órgãos de saúde para a adequação aos preceitos legais. Primeiro no treinamento, com déficit de profissionais especializados, escassos conhecimentos sobre as reais necessidades de cada local, e dificuldades de diálogo com o Ministério da Saúde para melhor compreensão do funcionamento da política. Segundo, no âmbito do acesso, com a demora no encaminhamento para tratamento e dificuldades na qualidade do atendimento com um possível aumento na demanda. Terceiro, a qualidade do tratamento oferecido pelo Sistema Único de Saúde e a falta de recursos financeiros. E, por fim, as dificuldades das próprias pacientes, com a demora na aceitação do diagnóstico e busca pelo tratamento.

O relatório destacava que embora a publicação da lei tenha sido um passo importante para melhorar o acesso e acompanhamento de pacientes com neoplasias no SUS, a sua plena implementação ainda não havia sido concluída. Dessa forma, era apontado um rol de melhorias para permitir que as pacientes realmente se beneficiassem das mudanças. Destas melhorias podemos destacar: aquisição de equipamentos e materiais, contratação e capacitação de profissionais, investimento financeiro, acesso à internet nas unidades básicas de saúde, e a efetiva utilização do SISCAN para encaminhamento e acompanhamento das pacientes (FEMAMA, 2014).

A FEMAMA também participou de Audiência Pública, realizada em 22 de maio de 2014, para discussão do primeiro ano de vigência da Lei 12.732/2012 e as dificuldades em sua implementação. A audiência ocorreu na Comissão de Assuntos Sociais do Senado, tendo sido requerida pela senadora Ana Amélia Lemos. Em sua fala, a senadora avaliou a aplicação da lei argumentando que, até aquela data, a legislação ainda não estava sendo contemplada em sua totalidade. Para total efetividade, Ana Amélia destacava que o Ministério da Saúde precisaria fazer ajustes na portaria que normatizava o texto legal de forma a desburocratizar a

\footnotetext{
${ }^{10}$ Informações disponíveis em http://www.otempocorrecontra.com.br. Acesso em 10 jan. 2021
} 
regulamentação. Segundo Ana Amélia, quanto mais burocracia, maior demora e menor efetividade. Ainda, com base na gestão pública, a atuação do governo federal auxiliaria a evitar possíveis ações de judicialização pelo não cumprimento da lei (FEMAMA, 2014).

Em relação às dificuldades na ampla implementação do marco legal, a presidente da FEMAMA, Maira Caleffi destacou em sua fala na Audiência citada que faltavam recursos no Sistema Único de Saúde, incluindo profissionais, elementos técnicos, dentre outros, para o início do tratamento de câncer e, consequentemente, a correta aplicação da lei. A presidente apresentou dados do relatório da pesquisa da campanha "O Tempo Corre Contra", que indicavam um delay entre diagnóstico e início de tratamento em uma média de 6 a 8 meses. Com base nos dados, Maira discutiu e criticou a consistência dos registros de câncer no país, apontando para a necessidade de completa implantação do SISCAM para uma real implementação da lei, tendo em vista se tratar de num país de dimensões sociais marcadas pela desigualdade (IDEM).

A médica destacou ainda que o câncer de mama era um dos tipos da doença com maiores possibilidades de cura, quando diagnosticado precocemente. Mas, por outro lado, ainda era um dos que mais levava a óbito. Por isso da relevância dos marcos legais para modernização e eficácia das ações de diagnóstico e tratamento. Maira argumentou ainda que, após o diagnóstico do câncer, a consulta com o médico especialista já era demorada. E, após essa primeira consulta, ainda eram necessários exames de estadiamento para definir as medidas de tratamento. Com isso, a delonga e dificuldades na aplicação da lei eram ainda maiores. Portanto, para o seu efetivo cumprimento, segundo a presidente da FEMAMA, era preciso garantir o amplo acesso do paciente ao sistema de saúde (IDEM).

Após as ações e pressões por parte principalmente da FEMAMA na articulação com o poder legislativo, o Ministério da Saúde publicou a Portaria 1.220/14 que alterou a portaria 876/13, reestabelecendo a contagem do prazo de 60 dias para início do tratamento de câncer na rede de saúde pública a partir da data do laudo patológico e não do registro no prontuário do paciente, reduzindo assim, o tempo de espera. No entanto, para que o tratamento fosse otimizado, o diagnóstico também deveria ser regulamentado de forma a garantir que "o tempo não corresse contra" a cura da paciente. Essa foi outra pauta de ação da FEMAMA, que analisaremos a seguir.

\section{Lei 13896/2019: "Lei dos 30 dias"}


Além da articulação nos debates da Lei 12.732/2012, a FEMAMA teve um papel importante de ativismo político em relação à Lei 13.896/2019, que postulava que, mediante suspeita de câncer, o laudo diagnóstico deveria estar concluído e disponibilizado à paciente em até 30 dias. Sobre a temática da lei, é importante destacar que a realização do diagnóstico exerce um papel central na definição da própria doença. É o ponto de articulação entre o médico e o paciente, sendo o mecanismo de comunicação e de identidade da enfermidade, pois é através dele que a mesma é especificada e ocorre um processo de legitimação tanto da prática médica quanto do papel da doença no meio social. $\mathrm{O}$ ato de diagnosticar estrutura a prática médica, conferindo aprovação social e definindo as relações entre doença, paciente e sociedade (ROSENBERG, 2002).

As discussões para a Lei 13896/2019 tiveram início em 2018, com o projeto de lei 143/2018 - "PCL dos 30 dias". O projeto previa que, mediante suspeita de câncer, a confirmação diagnóstica deveria ocorrer na rede pública de saúde em no máximo um mês. A atuação da FEMAMA na "Lei dos 30 dias" caminhou desde o lobby político para sansão da lei, passando pela inclusão da temática na pauta da campanha do Dia Mundial do Câncer de 2019.

Na campanha do Dia Mundial do Câncer de 2019, datado em 04 de fevereiro, a FEMAMA lançou em suas redes sociais a chamada: "Eu sou a FEMAMA e vou lutar pelo diagnóstico do câncer em 30 dias". A mensagem remetia à importância do diagnóstico para aumento das possibilidades de cura das pacientes. Simbolicamente, no dia da campanha, a Federação, através das suas filiadas, entregou ofícios aos senadores dos respectivos estados onde se fazem presentes, explicando a importância do projeto de lei e solicitando apoio parlamentar em sua tramitação. A justificativa era de que a demora no diagnóstico realizado no Sistema Único de Saúde reduzia as chances de cura das pacientes. Naquele contexto, a espera média pelo diagnóstico seria de 270 dias, o que impactava em mais de $55 \%$ dos casos identificados em estágio avançado de câncer (FEMAMA, 2020b).

A Lei foi aprovada no Senado Federal em 16 de outubro de 2019. E, para que tivesse efetividade, precisava ser sancionada. Foi nesse ponto que a ação da FEMAMA, de ativismo plítico, se fez de forma mais efetiva, através da presidente Maira Caleffi. A médica entregou ao então vice presidente da República, Hamilton Mourão, presidente em exercício na ocasião, um ofício pedindo sanção do projeto da "Lei dos 30 dias", em 31 de outubro de 2019, reforçando que a solicitação deveria ser atendida no mesmo dia da entrega, junto ao Ministro Chefe da 
Casa Civil. Isso porque, após a aprovação da lei no Senado, a sanção presidencial deveria ocorrer em até 15 dias $^{11}$.

Por conta dos prazos, para garantir que a lei fosse implementada e entrasse em vigor o quanto antes, a FEMAMA realizou ainda um grande chamado em suas redes sociais para angariar apoio à causa. A Federação criou um abaixo assinado junto ao "Movimento Todos juntos contra o Câncer" para mobilizar forças e conscientizar os líderes do poder executivo brasileiro sobre a importância da sanção dentro da Campanha Outubro Rosa de 2019. A lei, por sua vez, foi sancionada em 30 de outubro de 2019, como Lei 13.896/2019 .

No entanto, a luta política da FEMAMA não terminava com a sanção, pois a regulamentação pelo Ministerio da Saude deveria ocorrer até abril de 2020, o que só se efetivaria em julho do mesmo ano. A Lei, mesmo não regulamentada, tem sua existência e eficiência garantidas. No entanto, a não regulamentação pelo Ministério da Saúde traria dificuldades no acesso e na ampla efetividade da Lei. A regulamentação está associada a questões práticas, de exercer competências técnicas para o estabelecimento de parâmetros, programas, organização de políticas públicas, e estabelecimento do desenho institucional de programas e ações de acesso pelo Sistema Único de Saúde.

Após regulamentação da lei, a FEMAMA apontava ainda para os principais desafios para ampla implementação da mesma. Desses desafios estavam a importância de um mapeamento das pacientes com casos suspeitos para acompanhamento das etapas do diagnóstico, e a organização da rede pública de saúde para ação e acompanhamento da paciente. Em suma, a "lei dos 30 dias" estabelecia essa temporalidade como intervalo máximo entre a suspeita do médico até o resultado do diagnostico, passando a valer a partir daí a "Lei dos 60 dias”, para início efetivo do tratamento (FEMAMA, 2020b).

\section{Considerações finais}

Em fins dos anos 1990 surgiu no Brasil um novo modelo de associações de pacientes, enredado na nova configuração da saúde nacional e com preceitos políticos relacionados ao ativismo e ao advocacy. A FEMAMA, representante da organização e federalização desse movimento, foi organizada ligada a pautas políticas, tendo no ativismo a principal bandeira de atuação, na conjunção de esforços para formação de uma rede com associações parceiras e filiadas.

\footnotetext{
${ }^{11}$ Informações disponíveis em www.femama.org.br.

${ }^{12}$ Informações disponíveis em www.femama.org.br.
} 
Em relação ao câncer de mama, percebemos que as iniciativas de ativismos transformaram as formas pelas quais a doença foi entendida, estudada e experenciada. O papel da informação e conscientização foi crucial no empoderamento das pacientes, no apoio às famílias e nas perspectivas do tratamento, com informação e acesso aos diretos das pacientes nos sistemas de saúde. Nessa discussão, o conceito de biomedicalização nos permitiu analisar a ligação entre a emergência de novos grupos e identidades. O surgimento de novas formas de sociabilidade, de solidariedade e compartilhamento facilitaram as condições para o fortalecimento das associações de pacientes e as práticas de advocacy e ativismo político em relação ao câncer de mama.

O princípio do advocacy, como discutimos nesse artigo, emergiu de uma rediscussão entre o papel do Estado e da sociedade civil, preconizando ações individuais e de grupos organizados com o objetivo de influenciar autoridades e particulares para necessidades sociais. Em seu sentido mais amplo, o princípio se caracteriza por iniciativas de pressão política, de promoção e defesa de uma causa e/ou interesse, e de articulações mobilizadas por organizações da sociedade civil com o objetivo de ampliar a visibilidade a determinadas temáticas ou questões no debate público e influenciar políticas visando transformações na esfera social.

As associações de advocacy, como a FEMAMA, ao possibilitarem parcerias e alianças políticas entre diversos atores sociais, contribuíram para a garantia do direito das mulheres ao diagnóstico precoce do câncer de mama e melhor qualidade de vida para as que vivenciavam a experiência da doença. As ações em advocacy possibilitaram a criação de novos espaços de participação e mudanças de leis com impacto nas políticas públicas.

Dois aspectos foram centrais na atuação da FEMAMA: a estrutura do sistema de saúde e os interesses coletivos; e os direitos individuais das pacientes. A prática de advocacy possibilitou ampliar a conscientização sobre o câncer de mama como uma questão social e política e a luta pela garantia das pacientes pelo atendimento agilizado no sistema público de saúde. No caso da Federação, possibilitou ainda o fortalecimento do perfil de cada associada e da própria dinâmica do trabalho em rede. Nesse sentido, a atuação da FEMAMA como sociedade civil atuante em advocacy com o paciente, foi efetiva na aprovação e regulamentação dos marcos temporais dos principais fatores relacionados ao estadiamento da doença e diminuição das possibilidades de cura: o diagnóstico precoce e tratamento rápido.

Por fim, para além da atuação nas prerrogativas legais em relação a direitos de acesso e tratamento às pacientes com câncer de mama, a atuação da FEMAMA englobou iniciativas de educação e informação, tanto de caráter científico quanto psicológico, a pacientes e familiares. A atuação pública, na divulgação e circulação de informações e conhecimentos sobre a doença, 
direitos das pacientes e orientações a familiares, foi também uma grande frente de ação da FEMAMA. No domínio virtual da Federação foram e estão disponibilizadas orientações sobre caminhos de garantia dos direitos das pacientes com câncer de mama, tanto no atendimento do SUS, em requerimentos às secretarias de saúde e ações judiciais; quanto no atendimento conveniado em planos de saúde, com registros nos serviços de atendimento ao consumidor, reclamações às ouvidorias, a órgãos de defesa do consumidor e, ainda, ações judiciais.

A partir dos apontamentos aqui tecidos, podemos perceber que há um amplo espectro de questões que envolvem o câncer de mama no Brasil e sua história e ainda uma pluralidade de fontes de pesquisa a serem exploradas. Acreditamos que a discussão aqui apresentada possa fomentar interesses sobre a temática, possibilitando a ampliação de análises históricas sobre o câncer de mama, a atuação de associações de pacientes e as agendas públicas de assistência e controle de enfermidades, enriquecendo o debate mais geral sobre história das doenças e das políticas públicas em saúde no Brasil do século XX.

\section{Referências bibliográficas}

ANDREWS, Keneth; EDWARDS, Bob. Advocacy Organizations in the U.S. Political Process. Annual Review of Sociology. v. 30, p.479-506, 2004.

AVNER, Marcia. The Lobbying and Advocacy Handbook for Nonprofit Organizations: Shaping Public Policy at the State and Local Level. Minnesota: Amherst H. Wilder Foundation, 2002.

AZEVEDO e SILVA, et. al. "A situação dos cânceres do colo do útero e da mama no Brasil". In: TEIXEIRA, Luiz (org). Câncer de Mama e Colo de Útero: conhecimentos, políticas e práticas. Rio de Janeiro: Outras Letras, 2015, p. 41-74.

BARROS, Ângela Ferreira; UEMURA, Gilberto; MACEDO, Jefferson Lessa Soares. Atraso no diagnóstico e tratamento do câncer de mama e estratégias para a sua redução. FEMINA | Janeiro/Fevereiro 2012 | vol $40 \mid n^{\circ}$ 1, p. 31-36.

BRASIL. LEI No 13.896, DE 30 DE OUTUBRO DE 2019. Altera a Lei ${ }^{\circ} 12.732$, de 22 de novembro de 2012, para que os exames relacionados ao diagnóstico de neoplasia maligna sejam realizados no prazo de 30 (trinta) dias, no caso em que especifica. 2019.

BRASIL. Instituto Nacional de Câncer José Alencar Gomes da Silva. Diretrizes para a detecção precoce do câncer de mama no Brasil/ Instituto Nacional de Câncer José Alencar Gomes da Silva - Rio de Janeiro: INCA, 2015.

BRASIL. LEI No 12.732, DE 22 DE NOVEMBRO DE 2012. Dispõe sobre o primeiro tratamento de paciente com neoplasia maligna comprovada e estabelece prazo para seu início. 2012.

BRASIL. Ministério da Saúde. Secretaria de Atenção à Saúde. Instituto Nacional de Câncer. Controle de Câncer de Mama: documento de consenso. Brasília. Ministério da Saúde, 2004. CARNAVALLI, Keila de Moraes. A vida como palco de disputas: doença, ativismo social e processos comunicacionais em oncologia. 2021. Tese (Doutorado em Ciências) - Programa de Pós-Graduação stricto sensu em Informação e Comunicação em Saúde, FIOCRUZ. Rio de Janeiro, 2021. 
CLARKE Adele, et. al. Biomedicalization: Technoscientific Transformations of Health, Illness, and U.S. Biomedicine. American Sociological Review. 68(April): 161-194. 2003.

DALLARI, S. G. et al . Advocacia em saúde no Brasil contemporâneo. Rev. Saúde Pública, São Paulo, v. 30, n. 6, Dec.1996.

FEMAMA, 2020a. Lei dos 60 Dias: Tratamento dos pacientes com câncer. Disponível em: https://www.youtube.com/watch?v=pO4q3TZ9DW8. Acesso em 20 dezembro 2020.

FEMAMA 2020b. Lei dos 30 dias e como a COVID afeta o diagnóstico do câncer de mama. Disponível em: https://www.youtube.com/watch?v=TvUnXOSXsAM. Acesso em 10 dezembro 2020.

FEMAMA, 2018. Ciclo de Debates sobre Câncer de Mama para Parlamentares Minas Gerais, realizado em 20/06/2018, na Assembleia Legislativa de Minas Gerais, em Belo Horizonte (MG). Disponível em https://www.youtube.com/watch?v=sgyeig42gw0. Acesso em 5 janeiro 2021.

FEMAMA, 2014. Discurso Senadora Ana Amélia avaliando a aplicação da Lei dos 60 Dias, em sessão plenária do Senado Federal, em 22 de maio de 2014. Disponível em: https://www.youtube.com/watch?v=547H6EXY7vQ . Acesso em 12 janeiro 2021.

FEMAMA, 2013 A. Discurso de Maira Caleffi na audiência pública da Comissão de Assuntos Sociais (CAS) do Senado Federal em 21 de maio de 2013, para debater a regulamentação da Lei 12.732/12 . Disponível em: https://www.youtube.com/watch?v=KN2YlDBm9Uk. Acesso em 15 janeiro 2021.

FEMAMA. Pesquisa implementação da Lei 12.732/2012 - Relatório, 2014. Disponível em: http://www.otempocorrecontra.com.br/files/Pesquisa\%20Femama\%20Lei\%20dos\%2060\%20 Dias.pdf. Acesso em 10 janeiro 2021.

FEMAMA. \#OtempoCorreContra. Femamaorg, 50', 2013. Disponível em: https://www.youtube.com/watch?v=pVhaHkvC-hY. Acesso em 5 janeiro 2021.

ESTATUTO FEMAMA. Federação Brasileira de Instituições Filantrópicas de Apoio à Saúde da Mama - FEMAMA - Estatuto, 2010.

GARDNER, Kirsten E. Early Detection: Women, Cancer, and Awareness Campaigns in the Twentieth-Century United States. Chapel Hill: University of North Carolina Press, 2006. INCA. Instituto Nacional do Câncer José Alencar Gomes da Silva. Estimativa 2020: incidência de câncer o Brasil. Rio de Janeiro: INCA, 2019. Disponível em: https://www.inca.gov.br/sites/ufu.sti.inca.local/files//media/document//estimativa-2020-

incidencia-de-cancer-no-brasil.pdf. Acesso em 10 abr. 2020.

INCA. Instituto Nacional do Câncer José Alencar Gomes da Silva. Atlas de Mortalidade por Câncer. INCA, 2018. Disponível em: https://www.inca.gov.br/app/mortalidade. Acesso em 25 ago. 2020.

INCA. Instituto Nacional do Câncer José Alencar Gomes da Silva. O Controle do Câncer de Mama no Brasil: Trajetórias e Controvérsias / Instituto Nacional do Câncer José Alencar Gomes da Silva; Fundação Oswaldo Cruz. Rio de Janeiro: INCA; FIOCRUZ, 2017. Entrevista Maira Caleffi.

KLAWITER, Mater. The Biopolitics of Breast Cancer. Changing cultures of disease and activism. London: University of Minnesota Press, 2008.

LANA, Vanessa. A experiência mineira no controle do câncer do colo do útero em meados do século XX: o Hospital de Ginecologia de Belo Horizonte. História, Ciências, SaúdeManguinhos, v. 27, p. 1077-1095, 2020.

LANA, Vanessa. Organização da especialidade médica e controle do câncer do colo do útero no Brasil: o Instituto de Ginecologia do Rio de Janeiro em meados do século XX. História, Ciências, Saúde-Manguinhos, v. 23, p. 683-701, 2016.

MEDEIROS, Giselle Coutinho; BERGMANN, Anke; AGUIAR, Suzana Sales; THULER, Luiz Claudio Santos. Análise dos determinantes que influenciam o tempo para o início do tratamento 
de mulheres com câncer de mama no Brasil. Cad. Saúde Pública, Rio de Janeiro, 31(6):12691282, jun, 2015, p. 1269-1282.

MOFFETT, Jill. Moving beyond the ribbon. An examination of Breast Cancer Advocacy and Activism in the US and Canada. Cultural Dynamics. 15(3): 287 - 306, 2015.

PORTO, Marco Antônio; TEIXEIRA, Luiz Antônio; SILVA, Ronaldo Correa. "Aspectos históricos do controle do câncer de mama no Brasil". Revista Brasileira de Cancerologia, 2013; 59(3): 331-339.

POWELL, Walter W.; STEINBERG, Richard (ed.). The Nonprofit Sector: A Research Handbook. New Haven: Yale University Press, 2006.

RABEHARISOA, V. Experience, knowledge and empowerment: The Increasing Role of Patients Organizations in Staging, Weighting and Circulating Experience and Knowledge. State of the Art. In: AKRICH, M.; NUNES, J. A.; PATERSON, F.; RABEHARISOA, V. (Ed.). The dynamics of patient organizations in Europe. Paris: Presses de L'École de Mines, p. 133-82, 2008.

ROSE, Nikolas. A política da própria vida. Biomedicina, poder e subjetividade no século XXI. São Paulo: Paulus, 2013.

ROSENBERG, Charles. "The Tyranny of Diagnosis: Specific Entities and Individual Experience". In: The Milbank Quarterly. Vol. 80, number 2, 2002.

SILVA, Ronaldo Correa. "Mamografia e rastreamento mamográfico: o debate da detecção precoce do câncer de mama contextualizado para a realidade brasileira". In: TEIXEIRA, Luiz (org). Câncer de Mama e Colo de Útero: conhecimentos, políticas e práticas. Rio de Janeiro: Outras Letras, 2015, p. 165-210.

SOUZA, Camila Brandão; at. al. Estudo do tempo entre o diagnóstico e início do tratamento do câncer de mama em idosas de um hospital de referência em São Paulo, Brasil. Ciência \& Saúde Coletiva, 20(12):3805-3816, 2015, p. 3805-3816.

SULIK, Gayle and ZIERKIEWICZ, Edyta. Gender, Power, and Feminisms in Breast Cancer Advocacy: Lessons from the United States and Poland. Journal of Gender and Power. Vol. 1, N. 1, 2014.

TEIXEIRA, Luiz Antonio e FONSECA, Cristina Maria. De doença desconhecida a problema de saúde pública: o INCA e o controle do câncer no Brasil. 1. ed. Rio de Janeiro: Instituto Nacional do Câncer, 2007. 